\title{
AFRL-RY-WP-TR-2014-0256
}

\section{DOMAIN DERIVATIVES IN DIELECTRIC ROUGH SURFACE SCATTERING}

Saba Mudaliar

Antennas \& Electromagnetics Technology Branch

Multispectral Sensing \& Detection Division

JANUARY 2015

Interim Report

Approved for public release; distribution unlimited.

See additional restrictions described on inside pages

STINFO COPY

AIR FORCE RESEARCH LABORATORY SENSORS DIRECTORATE

WRIGHT-PATTERSON AIR FORCE BASE, OH 45433-7320

AIR FORCE MATERIEL COMMAND

UNITED STATES AIR FORCE 


\section{NOTICE AND SIGNATURE PAGE}

Using Government drawings, specifications, or other data included in this document for any purpose other than Government procurement does not in any way obligate the U.S. Government. The fact that the Government formulated or supplied the drawings, specifications, or other data does not license the holder or any other person or corporation; or convey any rights or permission to manufacture, use, or sell any patented invention that may relate to them.

This report is the result of contracted fundamental research deemed exempt from public affairs security and policy review in accordance with SAF/AQR memorandum dated 10 Dec 08 and AFRL/CA policy clarification memorandum dated 16 Jan 09. This report is available to the general public, including foreign nationals.

\section{AFRL-RY-WP-TR-2014-0256 HAS BEEN REVIEWED AND IS APPROVED FOR PUBLICATION IN ACCORDANCE WITH ASSIGNED DISTRIBUTION STATEMENT.}

*//Signature//

SABA MUDALIAR, Program Manager

Antenna \& Electromagnetic Technology Branch
//Signature//

TONY KIM, Chief

Antenna \& Electromagnetic Technology Branch

*//Signature//

TRACY W. JOHNSTON, Chief

Multispectral Detection \& Technology Division

Sensors Directorate

This report is published in the interest of scientific and technical information exchange, and its publication does not constitute the Government's approval or disapproval of its ideas or findings.

*Disseminated copies will show “//signature//” stamped or typed above the signature blocks. 


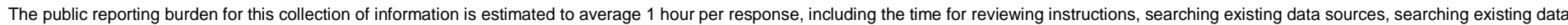

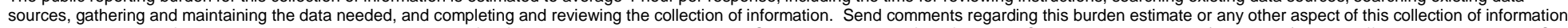

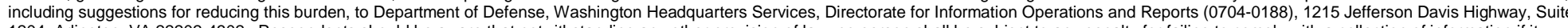

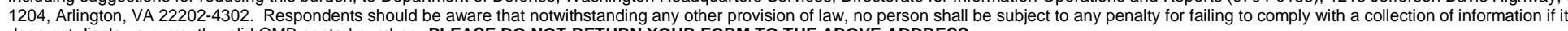
does not display a currently valid OMB control number. PLEASE DO NOT RETURN YOUR FORM TO THE ABOVE ADDRESS
1. REPORT DATE (DD-MM-YY)
January 2015
2. REPORT TYPE
Interim
3. DATES COVERED (From - To)
1 November 2012 - 30 September 2014

4. TITLE AND SUBTITLE

DOMAIN DERVATIVES IN DIELECTRIC ROUGH SURFACE SCATTERING

5a. CONTRACT NUMBER

In-house

5b. GRANT NUMBER

5c. PROGRAM ELEMENT NUMBER

61102F

6. AUTHOR(S)

Saba Mudaliar (AFRL/RYMH)

5d. PROJECT NUMBER

3001

5e. TASK NUMBER

13RY16COR

5f. WORK UNIT NUMBER

Y135

7. PERFORMING ORGANIZATION NAME(S) AND ADDRESS(ES)

Antennas \& Electromagnetics Technology Branch (AFRL/RYMH)

Multispectral Sensing \& Detection Division

8. PERFORMING ORGANIZATION REPORT NUMBER

AFRL-RY-WP-TR-2014-0256

Air Force Research Laboratory, Sensors Directorate

Wright-Patterson Air Force Base, OH 45433-7320

Air Force Materiel Command, United States Air Force

9. SPONSORING/MONITORING AGENCY NAME(S) AND ADDRESS(ES)

Air Force Research Laboratory

Sensors Directorate

Wright-Patterson Air Force Base, OH 45433-7320

Air Force Materiel Command

United States Air Force

10. SPONSORING/MONITORING AGENCY ACRONYM(S)

AFRL/RYMH

11. SPONSORING/MONITORING AGENCY REPORT NUMBER(S)

AFRL-RY-WP-TR-2014-0256

12. DISTRIBUTION/AVAILABILITY STATEMENT

Approved for public release; distribution unlimited

13. SUPPLEMENTARY NOTES

PAO Case Number 88ABW-2014-5990, Clearance Date 12 December 2014. Report contains color.

14. ABSTRACT

The inverse scattering solution of shape reconstruction is often posed as a problem in nonlinear minimization of an objective function with respect to $\mathrm{N}$ number of unknown model parameters characterizing the scatterer. The minimization procedures are usually iterative, and require the gradient of the objective function in the unknown model parameter vector at each stage of iteration. For large N, finite-differencing becomes numerically intensive, and an efficient alternative is domain differentiation in which the full gradient is obtained by solving a single scattering problem of an auxiliary field using the same scattering operator as that of the forward solution. This report presents the domain derivative calculation of the gradient for a locally perturbed dielectric interface. The method is non-variational, and algebraic in nature in that it evaluates the gradient by directly domain differentiating the scattering equations. The mathematical transformation of the scattering problem into the corresponding problem for the differentiated fields can be visualized explicitly. The formulation of and the motivation behind introducing the auxiliary field are explicitly demonstrated. Closed-form analytic expressions are obtained for the gradients for electromagnetic TE/TM scattering from dielectric rough surfaces, and for scalar wave scattering from Neumann and Dirichlet rough surfaces.

15. SUBJECT TERMS

scattering, locally perturbed, dielectric rough surface, domain differentiation, eulerian derivative

16. SECURITY CLASSIFICATION OF:

\begin{tabular}{|l|l|l|}
\hline a. REPORT & b. ABSTRACT & c. THIS PAGE
\end{tabular}

Unclassified Unclassified Unclassified
17. LIMITATION OF ABSTRACT: SAR
18. NUMBER OF PAGES 32 19a. NAME OF RESPONSIBLE PERSON (Monitor)

Saba Mudaliar

19b. TELEPHONE NUMBER (Include Area Code) $\mathrm{N} / \mathrm{A}$ 


\section{Table of Contents}

Section $\quad$ Page

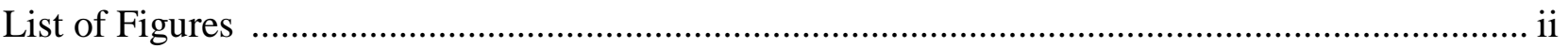

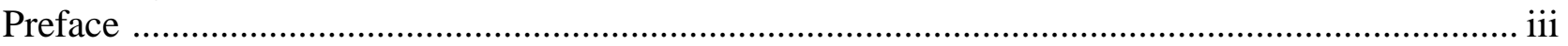

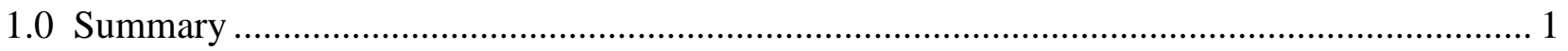

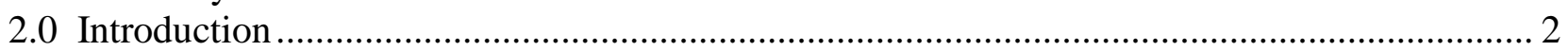

3.0 Some Relevant Identities from Domain Differentiation ................................................. 6

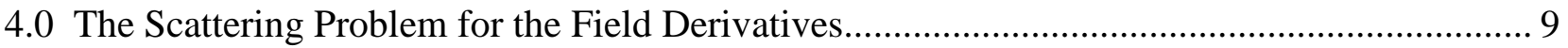

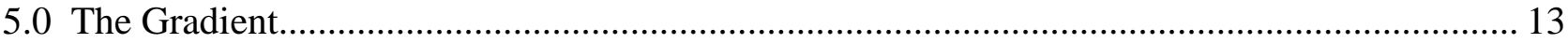

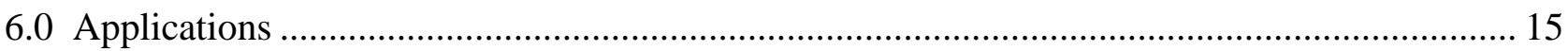

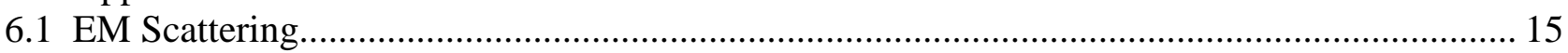

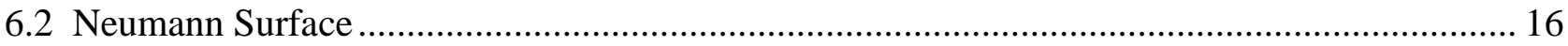

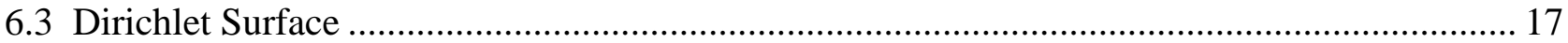

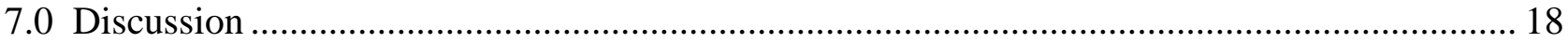

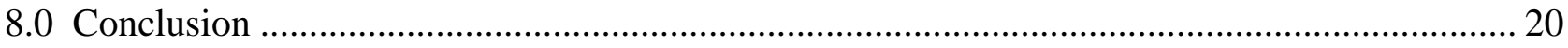

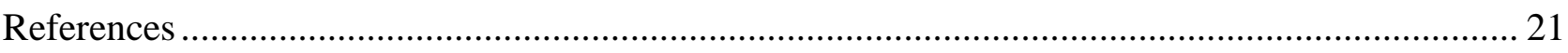

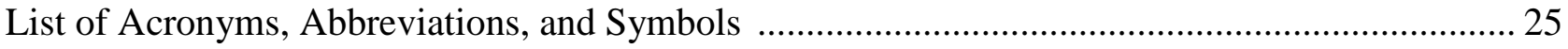




\section{List of Figures}

\section{Figure}

Page

1. A Schematic Illustration of the Scattering Geometry

2. Domain Variation of a Compactly Supported Scatterer ...................................................... 6

3. Domain Variation of a Locally Perturbed Surface .................................................................. 10

4. Convention of Vector Orientation for TE and TM Scattering ............................................... 15 


\section{PREFACE}

The author thanks Dr. Dilip Ghosh Roy for his work in this project while he was an on-site contractor during FY13. 


\section{Summary}

The inverse scattering solution of shape and/or material parameter reconstruction is often posed as a problem in nonlinear minimization of an objective function with respect to $N$ (usually large) number of unknown model parameters characterizing the scatterer. The minimization procedures are usually iterative, and require the gradient of the objective function in the unknown model parameter vector in each stage of iteration. For large $N$, finite-differencing becomes numerically intensive, and an efficient alternative is domain differentiation in which the full gradient is obtained by solving a single scattering problem of an auxiliary field using the same scattering operator as that of the forward solution. A well-known technique in this direction is the so-called adjoint field method which obtains the gradient by variationally minimizing an augmented objective function that includes the reduced wave equation via a Lagrange multiplier. Results are reported mostly for compact objects. This report presents the domain derivative calculation of the gradient for a one-dimensional, locally perturbed, infinitely long dielectric interface. The method is non-variational, and algebraic in nature in that it evaluates the gradient by directly domain differentiating the scattering equations. The computations are straightforward, and easy to follow. The mathematical transformation of the scattering problem into the corresponding problem for the differentiated fields can be visualized explicitly. The formulation of and the motivation behind introducing the auxiliary field are explicitly demonstrated. Closed-form analytic expressions are obtained for the gradients for electromagnetic transverse electric / transverse magnetic (TE/TM) scattering from dielectric rough surfaces, and for scalar wave scattering from Neumann and Dirichlet rough surfaces. Results compared with those for compact scatterers. Finally, the relationship between our results and Lorentz reciprocity is pointed out and clarified. 


\section{Introduction}

Consider the scattering of an electromagnetic field from a one-dimensional, infinitely long, locally perturbed, flat interface, $\Gamma$, shown in Figure 1 . It is assumed that the interface is located at $x_{2}=0$, and is invariant along $x_{3}$. The graph of the perturbation, $\gamma=\left\{x_{1}, x_{2}=f\left(x_{1}\right)\right\},-a \leq x_{1} \leq a$, is assumed to be smooth. Moreover, the profile, $f\left(x_{1}\right)$ is a single-valued function of $x_{1}$. The surface is thus not re-entrant. $\Gamma$ separates $R^{2}$ into an upper half-plane, $\Omega^{+}=\left\{x_{1}, x_{2}>f\left(x_{1}\right)\right\}$, and a lower half-plane, $\Omega^{-}=\left\{x_{1}, x_{2}<f\left(x_{1}\right)\right\}$, with wavenumbers, $k^{+}, k^{-}$, and dielectric constants, $\epsilon^{+}$and $\epsilon^{-}$, respectively. It is further assumed that the perturbed surface is illuminated at a non-grazing angle $\left(\left|\theta^{i n c}\right|<\pi / 2\right)$ by a beam $\left(\psi^{\text {inc }}\right)$ of finite width, e.g., a beam with a Gaussian angular spectrum [40] or a tapered Thorsos beam [44] having negligible energy beyond $\left|x_{1}\right| \geq A$, with $A \gg a$. Finite width beams and their properties are discussed in [23, 45, 24].

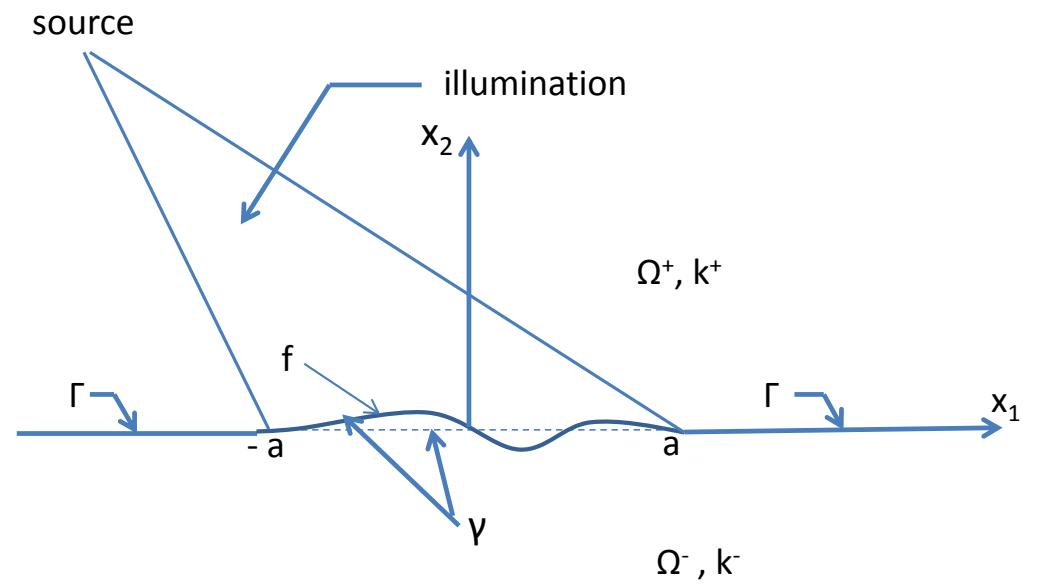

Figure 1: A Schematic Illustration of the Scattering Geometry

The following equations $[45,37,34,32,8,38,41,28]$ describe the scattering problem.

$$
\begin{aligned}
\left(\mathcal{L}^{+} \psi^{+}\right)(\vec{x}) & =0, \vec{x} \in \Omega^{+}, \\
\left(\mathcal{L}^{-} \psi^{-}\right)(\vec{x}) & =0, \vec{x} \in \Omega^{-}, \\
\psi^{+} & =\psi^{-}, \vec{x} \in \Gamma, \\
\alpha^{-} \psi_{, n}^{+} & =\alpha^{+} \psi_{, n}^{-}, \vec{x} \in \Gamma .
\end{aligned}
$$

In Eqs. (1)-(4), $\mathcal{L}^{ \pm}=\Delta+\left(k^{ \pm}\right)^{2}, \Delta$ being the Laplacian, and a time harmonicity of $e^{-i \omega t}$ is assumed. $\psi^{ \pm}$are the total fields, and $\psi_{, n}^{ \pm}$their normal derivatives on the boundary. The unit normal, $\hat{n}$, is given by

$$
\hat{n}=\frac{1}{\sqrt{1+\left(f^{\prime}\left(x_{1}\right)\right)^{2}}}\left[f^{\prime}\left(x_{1}\right), 1\right]^{T},
$$


and points into $\Omega^{+} \cdot f^{\prime}$ is the derivative of $f$ in $x_{1}$, and the superscript $T$ denotes transpose. Moreover, $\psi^{+}=\psi^{i n c}+\psi^{s c}$ is the sum of the incident $\left(\psi^{i n c}\right)$ and scattered $\left(\psi^{s c}\right)$ field. For the scattering geometry in Figure 1, $\psi^{s c}$ satisfies $[34,10,9,2]$ the radiation condition at infinity [7]. The parameters, $\alpha^{ \pm}$, depend on the material constants in $\Omega^{ \pm}$, and the scattering process. Applications to electromagnetic TE and TM scattering from a dielectric surface, and for scalar wave scattering from Neumann and Dirichlet surfaces, are considered later.

For an arbitrary surface, a closed form solution of (1)-(4) is not expected. Several approximate asymptotic techniques have been developed during the past few decades for this kind of problems. Two classical approaches are the small perturbation method (SPM) $[39,3,46]$, and the Kirchhoff approximation (KA) [4, 3, 46]. SPM may be used when the surface fluctuations are small and smooth. KA is applicable for problems where the radii of fluctuations are large compared to the signal wavelength. One observes that SPM leads to a linear relationship between the scattered field and the function describing the surface fluctuation. Hence one can readily employ the Fourier transform technique to invert the scattered field to reconstruct the rough interface [48], [1]. It turns out that even when using KA, the scattered fields may be manipulated such that the the rough interface can be reconstructed using the Fourier transform technique [47], [42], [30]. These classical approaches to rough surface scattering, viz., SPM and KA, are single scattering approximations and hence a linear relationship between the scattered field and the underlying rough surface is possible. However if the surface roughness is not small and smooth, multiple scattering becomes important. Such processes are nonlinear in character and hence the solution to (1)-(4) is formally expressed as: $\psi^{s c}=\mathcal{F}(f)$, where $\mathcal{F}$ is a nonlinear operator that transforms the boundary, $\Gamma$, into scattered fields. The determination of $\psi^{s c}$, given $\mathcal{F}$ and $f$, constitutes the direct or forward problem. The inverse problem is to reconstruct the surface profile, $f$, where $\mathcal{F}$ and $\psi^{s c}$ are known. Formally, the inverse solution is: $f=\mathcal{F}^{-1}\left\{\psi^{s c}\right\}$, the result of applying the inverse scattering operator, $\mathcal{F}^{-1}$, to the data, $\left\{\psi^{s c}\right\}$. However, because of the nonlinear nature of $\mathcal{F}$, the inverse problem is frequently solved $[7,15,16]$ by transforming it into a problem in nonlinear optimization. The solution is obtained as the minimizer of an objective function, $\mathcal{J}(f)$, defined as:

$$
\begin{array}{r}
\mathcal{J}(f):=\frac{1}{2} \sum_{m=1}^{M}\left|\Delta^{\delta} \psi\left(\vec{x}_{m}\right)\right|^{2} \\
=\frac{1}{2} \sum_{m=1}^{M}\left(\Delta^{\delta} \psi\right)^{*}\left(\vec{x}_{m}\right)\left(\Delta^{\delta} \psi\right)\left(\vec{x}_{m}\right) .
\end{array}
$$

In (5), $\Delta^{\delta} \psi\left(\vec{x}_{m}\right):=\psi^{p r}\left(\vec{x}_{m}\right)-\psi^{\text {meas }}\left(\vec{x}_{m}\right)$ is the data misfit function, i.e., the difference between the measured $\left(\psi^{\text {meas }}\right)$ and a theoretically predicted scattered field $\left(\psi^{p r}\right)$, both at a detector location, $\vec{x}_{m}$. The complex conjugation is denoted by $*$, and $M$ is the total number of the detectors. The superscript, $\delta$, on $\Delta^{\delta}$ indicates that $\psi^{\text {meas }}$ is noisy, $\delta$ being 
the level of the measurement noise. Furthermore, $\psi^{\delta} \rightarrow \psi^{\text {exact }}$ as $\delta \rightarrow 0[13,27]$, where $\psi^{\delta}$ is a regularized solution.

Let $\vec{p}=\left\{p_{i}\right\}_{1}^{N}$ be the parametrization of $\gamma$. The inverse solution is then given by: $\vec{p}=\operatorname{argmin}_{X_{p}} \mathcal{J}(\vec{p}), X_{p}$ being the space of admissible parameters. The inverse problem being ill-posed, regularization [13] is necessary in order to obtain a meaningful solution. The standard procedures for the minimization of (5) are iterative in nature, and require that the gradient of the objective function (or the scattered fields) in the unknown model parameter vector be obtained in each stage of iteration. Let this derivative of $\mathcal{J}(f)$ be denoted by $\underline{d} \mathcal{J}(f)$.Then:

$$
\underline{d} \mathcal{J}(f)=-\operatorname{Re} \sum_{m=1}^{M}\left(\Delta^{\delta} \psi\right)^{*}\left(\vec{x}_{m}\right) \psi^{s \prime}\left(\vec{x}_{m}\right) .
$$

$\psi^{s \prime}$ in (6) is the domain derivative $[26,18,19]$, of $\psi^{s c}$, that is, the derivative of the scattered field with respect to the variation in $f$. Equation (6) can also be written as:

$$
\underline{d} \mathcal{J}(f)=-\operatorname{Re}\left\{J^{T}\left(\Delta^{\delta} \psi\right)^{*}\right\},
$$

where $J^{T}$ is the transpose of the Jacobian, $J$.

The procedure most often employed in inverse problems for the computation of derivatives of the cost function w.r.t. parameters of the problem is numerical [17]. For instance, in our problem, one can use a $N$ parameter model to represent the rough interface $\gamma$ and employ finite difference method to compute the domain derivative. This is a very general method applicable any complex problem. However, differentiating $\psi^{s c}$ with respect to a parameter vector of length $N$ involves solving $N$ distinct scattering problems for $N$ distinct scatterers. If $N$ is large (which is often the case in practice), then clearly, the finite-difference method becomes numerically intensive, besides accumulating large round-off errors. It essentially involves the calculation of the large Jacobian matrix, J. However, (7) shows that the evaluation of the derivative involves evaluating the product term, $J^{T}\left(\Delta^{\delta} \psi\right)^{*}$, and not the Jacobian explicitly. In the adjoint field method $[31,12,11,35,36,43,22,14]$, this product is obtained as the solution of the forward problem in Eqs. (1)-(4), but with a source term as in the R. H. S. of (6). The source is obtained by backpropagating the data misfit functions to the scatterer space. The boundary value problem for the adjoint field is derived by applying variational techniques to minimize an augmented objective function, namely, $\mathcal{J}$ in (5) plus a constraint term involving the reduced wave equation via Lagrange multiplier. The upshot of all this is that the entire gradient is evaluated by solving only one single scattering problem in which the same operator as that of the forward scattering problem is used. An important feature of this method is that it is essentially independent of $N$. There are two different ways one can carry out the domain derivative calculation using the adjoint method. Depending on the numerical procedure used for solving the forward problem one can use appropriate 
discretization and carry out the numerical calculation of the derivatives. This is a relatively more general procedure. In some problems it may be possible to obtain analytical closed-form expression for the domain derivative. This not only offers valuable physical insight into the problem but also gives the flexibility in the choice of algorithm used for shape reconstruction.

In this report, we focus attention on obtaining closed-form expression for the domain derivatives. There are several distinguishing features in the calculations of this report. In the literature we notice that domain derivatives of the scattered fields have been calculated mostly for compact scatterers, i.e., scatterers occupying a finite volume of space. In this report, on the other hand, an infinitely long, locally perturbed, dielectric interface is considered. The boundary variation in this case is relatively more subtle than that for a compact, volumetric object. Notice that we use neither variational procedures nor an augmented objective function in this report. The calculations are purely algebraic in nature in that the scattering equations are directly domain-differentiated without any intervening mathematical formalism. In this manner of presentation, the calculational procedures are found to be straightforward, physically transparent, and easy to follow. Moreover, the details of the mathematical transformation of the scattering problem into the corresponding problem for the differentiated fields can be traced explicitly. The present calculations also invoke an auxiliary scattering problem, as in the adjoint field case. However, the mathematical formulation of and the motivation behind the auxiliary problem are more transparent here than in the adjoint field calculations where the problem is a result of the mathematical manipulations. Also, the gradients are calculated for the electromagnetic TE and TM scattering from a dielectric rough surface as also for scalar wave scattering from a Neumann and a Dirichlet surface. Finally, the relation of the results thus obtained with the Lorentz reciprocity is discussed and clarified.

The report is organized as follows. Some important relations from domain differential calculus, relevant to the present calculations, are summarized in Section 3. These are then used in Section 4 to derive the boundary value problem for the derivative fields, $\psi^{ \pm \prime}$, of $\psi^{ \pm}$. The differential, $\underline{d} \mathcal{J}(f)$, and the gradient, $\nabla_{f} \mathcal{J}(f)$, of $\mathcal{J}(f)$, are evaluated in Section 5. The method is next applied to electromagnetic TE and TM scattering, and Neumann and Dirichlet boundary conditions in Section 6. Discussions are presented in Section 7, followed by a summary in Section 8 . 


\section{Some Relevant Identities From Domain Differentiation}

Let $\mathcal{U}$ be a piecewise smooth bounded open region in $R^{2}$ with boundary $\Sigma$. A continuously differentiable vector field, $\vec{V}$, is defined in $\mathcal{U}$. It is assumed that $\vec{V}$ or its normal derivative vanishes on $\Sigma$. Consider the mapping, $T_{\tau}(\vec{V}(\vec{x}))=I+\tau \vec{V}(\vec{x})+O\left(\tau^{2}\right), \vec{x} \in \mathcal{U}$, continuously mapping $\mathcal{U}$ onto itself. $I$ is the identity operator, and the parameter, $\tau \in[0, t], t \in R^{1} . T_{\tau}$ introduces a change of variable from $\vec{x} \rightarrow \vec{x}_{\tau}=\vec{x}+\tau \vec{V}(\vec{x})+O\left(\tau^{2}\right)$. Let $\Omega \subset \mathcal{U}$ be a domain in $\mathcal{U}$. Then $T_{\tau}(\Omega)=\Omega_{\tau}$, where $\Omega_{\tau}=\left\{\vec{x}_{\tau} \mid \vec{x}_{\tau}=\vec{x}+\tau \vec{V}(\vec{x}), \forall \vec{x} \in \Omega\right\}$. Moreover, $\Omega_{\tau} \subset \mathcal{U}, \forall \tau \in[0, t] . \tau$ defines the magnitude of the deformation. In fluid dynamical problems, $\tau$ plays the role of the time variable. These are illustrated in Figure 2. It can be shown [49] that for small $\tau, T_{\tau}$ is a diffeomorphism in $\mathcal{U}$, that is, a bijection with a continuous inverse, with same degree of smoothness as the vector field. This inverse is given by: $T_{\tau}^{-1}(\vec{V})=I-\tau \vec{V}+O\left(\tau^{2}\right)$.

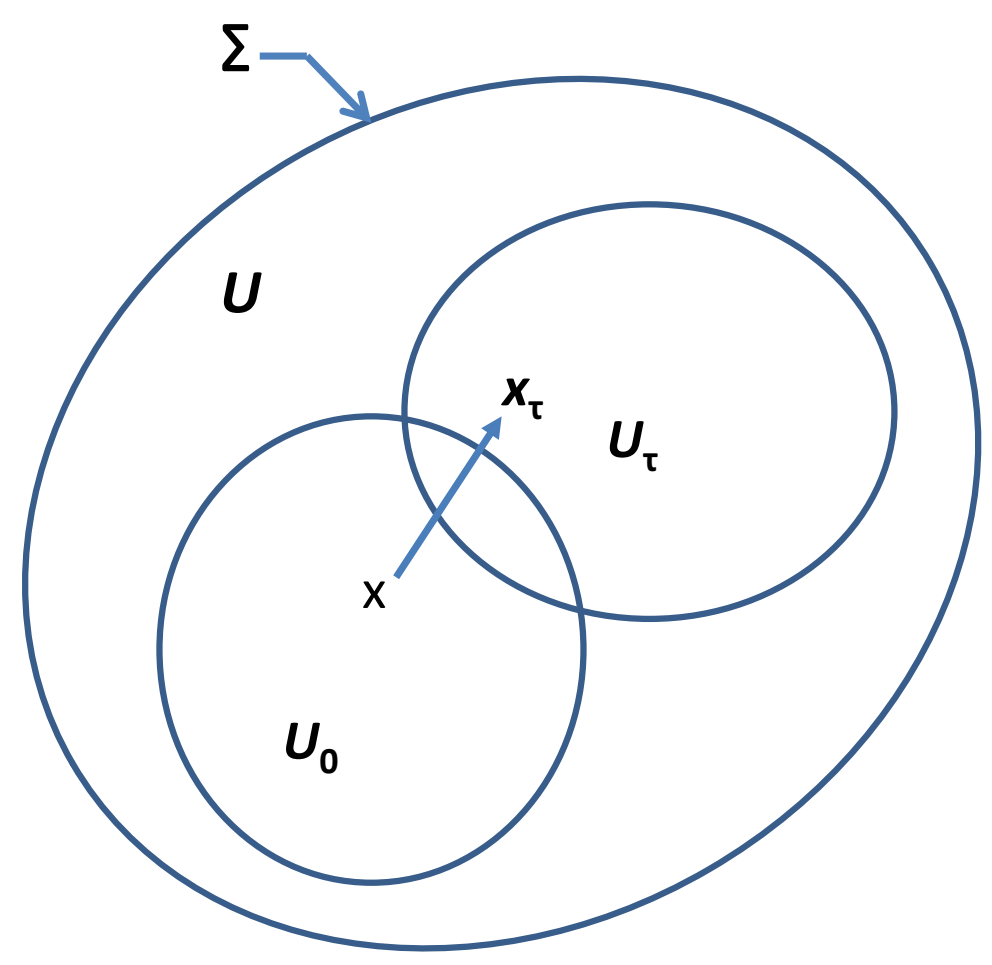

Figure 2: Domain Variation for a Compactly Supported Scatterer

Let $\phi(\vec{x})$ be the solution of a boundary value problem defined on $\Omega$, which is considered to be the unperturbed domain, i.e., $\Omega=\Omega_{\tau=0}$. Let $T_{\tau}$ transform $\Omega$ to the perturbed domain, $T_{\tau}(\Omega)=\Omega_{\tau}$, and let $\phi_{\tau}\left(\vec{x}_{\tau}\right), \vec{x}_{\tau} \in \Omega_{\tau}$, be the corresponding solution, now defined on $\Omega_{\tau}$. Note that $\tau$ appears twice in $\phi_{\tau}\left(\vec{x}_{\tau}\right)$, once as a subscript in $\phi_{\tau}$, and second time in 
$\vec{x}_{\tau}$, which is a running variable. It is the dependence of $\phi$ on $\tau$ that gives rise to domain differentiation in place of the regular, partial derivatives. The Eulerian (also called total or material) derivative of $\phi$ at $\vec{x}$, denoted by $\dot{\phi}(\vec{x})$, is defined as:

$$
\dot{\phi}(\vec{x})=\lim _{\tau \rightarrow 0} \frac{1}{\tau}\left[\phi_{\tau}\left(\vec{x}_{\tau}\right)-\phi(\vec{x})\right]
$$

The derivative in (8) can also be written as the directional derivative:

$$
\begin{aligned}
\dot{\phi}(\vec{x}) & =\left.\frac{d}{d \tau}\left(\phi \cdot T_{\tau}\right)\right|_{\tau=0} \\
& =\left.\frac{d}{d \tau} \phi_{\tau}\left(\tau, \vec{x}_{\tau}\right)\right|_{\tau=0}
\end{aligned}
$$

$\dot{\phi}$, as defined in (8) (or (9)), is a Gateaux differential [29], which is defined in a pointwise sense at $\vec{x} \in \Omega$. Equation (9) can be further expressed as:

$$
\begin{aligned}
\dot{\phi}(\vec{x})= & \lim _{\tau \rightarrow 0} \frac{1}{\tau}\left[\phi_{\tau}\left(\vec{x}_{\tau}\right)-\phi(\vec{x})\right], \\
= & \lim _{\tau \rightarrow 0} \frac{1}{\tau}\left[\phi_{\tau}(\vec{x})-\phi(\vec{x})\right] \\
& +\lim _{\tau \rightarrow 0} \frac{1}{\tau}\left[\phi_{\tau}\left(\vec{x}_{\tau}\right)-\phi_{\tau}(\vec{x})\right] .
\end{aligned}
$$

The solution is assumed to be defined in $\mathcal{U} \times I \subset R^{n+1}, I=[0, t] \subset R^{1}$. $\phi_{\tau}$ is then considered to be the restriction of the solution defined on $\Omega_{\tau}$. Provided that the limit on the first term in the R. H. S. in (10) exists, the equation can be recast as:

$$
\dot{\phi}(\vec{x})=\phi^{\prime}(\vec{x})+\vec{V}(\vec{x}) \cdot \nabla \phi(\vec{x})
$$

where

$$
\phi^{\prime}(\vec{x})=\lim _{\tau \rightarrow 0} \frac{1}{\tau}\left[\phi_{\tau}(\vec{x})-\phi(\vec{x})\right]
$$

is the partial derivative of $\phi$ at $\vec{x}$, i.e., the derivative when $\vec{x}$ is held fixed. The term, $\vec{V} \cdot \nabla \phi$, is the convective part. Furthermore, since $\tau$ and $\vec{x}$ are independent variables, $\partial_{\tau}$ and $\partial_{x}$ commute, yielding the following important relation:

$$
(\nabla \phi)^{\prime}=\nabla \phi^{\prime}
$$

The Eulerian derivative of a domain functional can be obtained from (12). Let

$$
F\left(\phi_{\tau} ; \Omega_{\tau}\right)=\int_{\Omega_{\tau}} \phi_{\tau}\left(\vec{x}_{\tau}\right) d \vec{x}_{\tau}
$$

be a domain functional of $\phi_{\tau}$ over the perturbed domain, $\Omega_{\tau}$. It can be written as:

$$
F\left(\phi_{\tau} ; \Omega\right)=\int_{\Omega} \phi_{\tau}(\vec{x}+\tau \vec{V}(\vec{x}))\left|J_{T}\right| d \vec{x}
$$


$\left|J_{T}\right|$ is the Jacobian matrix of $T_{\tau}$, and $\left(J_{T}\right)_{i j}=\delta_{i j}+V_{i, j}, V_{i, j}=\partial V_{i} / \partial x_{j}$. Direct calculation yields: $\left|J_{T}\right|=\nabla \cdot \vec{V}$. Moreover, $\left.J_{T}\right|_{\tau=0}=I$. Then differentiating Eq. (14) in $\tau$ yields the Eulerian derivative, $\underline{d} F$, namely

$$
\underline{d} F(\phi ; \Omega)=\int_{\Omega} \phi^{\prime}(\vec{x}) d \vec{x}+\int_{\Gamma} \phi(\vec{x}) v(\vec{x}) d s .
$$

$v=\vec{V} \cdot \hat{n}$ is the normal component of $\vec{V}$ on $\Gamma$. It is demonstrated (Section 5$)$ that $\underline{d} F(\phi ; \Omega)$ is linear in the perturbation. The Gateaux derivative [29], therefore, exists, and $F(\phi ; \Omega)$ has a gradient.

Let us further introduce the following identities.

$$
\begin{gathered}
\nabla \phi=\nabla_{\Gamma} \phi+\hat{n}(\hat{n} \cdot \nabla \phi)=\nabla_{\Gamma} \phi+\hat{n} \phi_{, n}, \\
\nabla_{\Gamma} \phi \cdot \nabla_{\Gamma} u=\nabla \phi \cdot \nabla u-\phi_{, n} u_{, n}, \\
\int_{\Gamma} \nabla_{\Gamma} \cdot\left(z \nabla_{\Gamma} \phi\right) w d s=-\int_{\Gamma} z\left(\nabla_{\Gamma} \phi \cdot \nabla_{\Gamma} w\right) d s .
\end{gathered}
$$

$\nabla_{\Gamma}=\nabla-\hat{n} \partial_{n}$ is the surface gradient, and the functions $w$ and $z$ are defined in a neighborhood of $\Gamma$. Eq. (17) is sometime referred to as Stokes'identity which can be established using differential geometry [33]. Suffice it to say that the surface gradient of a function $u$ defined on the boundary is the restriction to the boundary of the lifting of $u$ defined in the tubular covering of the boundary. Finally, we introduce Green's second theorem $[25,7]$, which is:

$$
\int_{\Omega}[\phi \Delta \psi+\nabla \phi \cdot \nabla \psi] d \vec{x}=\int_{\Gamma} \phi \psi_{, n} d s .
$$

The unit normal, $\hat{n}$, is directed in the exterior of the domain $\Omega . \phi_{\tau}\left(\vec{x}_{\tau}\right)$ is a domain functional.

The above discussions were for a closed, bounded domain (Figure 2). In the case of the locally perturbed interface in Figure 1, the deformation is modified to that shown in Figure 3. As earlier, the velocity field is assumed to vanish on $\Sigma$. The surface profile, $f\left(x_{1}\right)$, separates $\mathcal{U}$ into $\mathcal{U}^{+}$and $\mathcal{U}^{-}$. Thus $\mathcal{U}=\mathcal{U}^{+}+\mathcal{U}^{-}$. As the curve $f\left(x_{1}\right)$ is deformed into $f_{\tau}\left(x_{1}\right), \mathcal{U}^{ \pm}$are deformed to $\mathcal{U}_{\tau}^{ \pm}$, but the overall domain, $\mathcal{U}$, remains unchanged. The relations which were derived above, must now be applied to the regions, $\mathcal{U}_{\tau}^{+}$and $\mathcal{U}_{\tau}^{-}$. All derivations assume $C^{2}$-smoothness of the boundary function. Thus $\psi^{ \pm} \in C^{2}\left(\bar{\Omega}^{ \pm}\right)$, and the deformation operator, $T_{\tau}$ is $C^{2}$-diffeomorphic. Furthermore, it is known that for the smoothness condition assumed here, the solutions can be extended to the neighborhood of the boundaries of $\Omega$, and $\Omega_{\tau}$. For details, we refer to [20]. We also note that the application of Green's theorem to $\mathcal{U}$ in Figure 3 may be problematic. However, the volume integrals involving $\Delta \psi^{ \pm}$, the Laplacian appears as being multiplied by functions that are smooth in the domain, and vanishes on the boundary. The application of Green's theorem then does not cause problems. Next we derive the BVP for the domain derivatives of $\psi^{ \pm}$in Eqs. (1)-(4) using the relations that were presented in this section. 


\section{The scattering problem for the field derivatives}

In this Section, we determine how the scattering equations (1)-(4) for the unperturbed surface are transformed as the surface is deformed by $T_{\tau}$. Scattering in the transformed domains, $\mathcal{U}^{ \pm}$, is described by:

$$
\begin{gathered}
\left(\mathcal{L}_{\tau}^{+} \psi_{\tau}^{+}\right)\left(\vec{x}_{\tau}\right)=0, \vec{x}_{\tau} \in \mathcal{U}_{\tau}^{+}, \\
\left(\mathcal{L}_{\tau}^{-} \psi_{\tau}^{-}\right)\left(\vec{x}_{\tau}\right)=0, \vec{x}_{\tau} \in \mathcal{U}_{\tau}^{-}, \\
\psi_{\tau}^{+}=\psi_{\tau}^{-}, \vec{x}_{\tau} \in \gamma_{\tau}, \\
\alpha^{-} \psi_{\tau, n}^{+}=\alpha^{+} \psi_{\tau, n}^{-}, \vec{x}_{\tau} \in \gamma_{\tau} .
\end{gathered}
$$

The Helmholtz equations (19) and (20) are transformed first. The transformation of the boundary conditions in Eqs. (21) and (22) follows next. From the discussion at the end of the previous Section, $\Gamma_{\tau}=\gamma_{\tau}$, and all differentiations are, therefore, with respect to $\gamma$. Taking the derivatives of Eqs. (19) and (20), and using (11) yields:

$$
\left(\Delta \psi^{ \pm}\right)^{\prime}+\vec{V} \cdot \nabla\left(\Delta \psi^{ \pm}\right)+\left(k^{ \pm}\right)^{2}\left(\left(\psi^{ \pm}\right)^{\prime}+\vec{V} \cdot \nabla\left(\psi^{ \pm}\right)\right)=0
$$

Now, $\left(\Delta \psi^{ \pm}\right)^{\prime}=\Delta\left(\psi^{ \pm}\right)^{\prime}$ by Eq. (13), and $\mathcal{L}^{ \pm} \psi^{ \pm}=0$ in $\mathcal{U}^{ \pm}$by virtue of Eqs. (1) and (2). It then follows that:

$$
\begin{aligned}
& \left(\mathcal{L}^{+} \psi^{+\prime}\right)(\vec{x})=0, \vec{x} \in \mathcal{U}^{+}, \\
& \left(\mathcal{L}^{-} \psi^{-\prime}\right)(\vec{x})=0, \vec{x} \in \mathcal{U}^{-}
\end{aligned}
$$

Now, in the exterior of $\mathcal{U}, \dot{\psi}^{ \pm}=\psi^{ \pm \prime}$, since the observation point is held fixed. The convective term then vanishes, and $\mathcal{L}^{ \pm} \psi^{ \pm \prime}$ vanishes outside $\mathcal{U}$. Therefore,

$$
\begin{aligned}
& \mathcal{L}^{+} \psi^{+\prime}(\vec{x})=0, \vec{x} \in \Omega^{+}, \\
& \mathcal{L}^{-} \psi^{-\prime}(\vec{x})=0, \vec{x} \in \Omega^{-} .
\end{aligned}
$$

Exactly similar considerations are applied to the transformation of the first boundary condition in Eq. (21). The result is:

$$
\begin{aligned}
\psi^{+\prime}-\psi^{-\prime} & =-\vec{V} \cdot \nabla\left(\psi^{+}-\psi^{-}\right) \\
& =-\vec{V} \cdot \nabla_{\gamma}\left(\psi^{+}-\psi^{-}\right)-v\left(\psi_{, n}^{+}-\psi_{, n}^{-}\right), \\
& =(\alpha-1) \psi_{, n}^{+} v .
\end{aligned}
$$

In Eq. (27), $\nabla_{\gamma}$ is the tangential derivative on $\gamma$. Furthermore, $\alpha=\alpha^{-} / \alpha^{+}$, and the boundary conditions in Eqs. (3) and (4) were used in arriving at the result.

The Eulerian derivative of the second boundary condition in Eq. (4) is calculated as follows. First we introduce the following notations.

$$
\int_{V^{ \pm}}^{ \pm} \Phi^{ \pm} d \vec{x}=\int_{V^{+}} \Phi^{+} d \vec{x} \pm \int_{V^{-}} \Phi^{-} d \vec{x}
$$




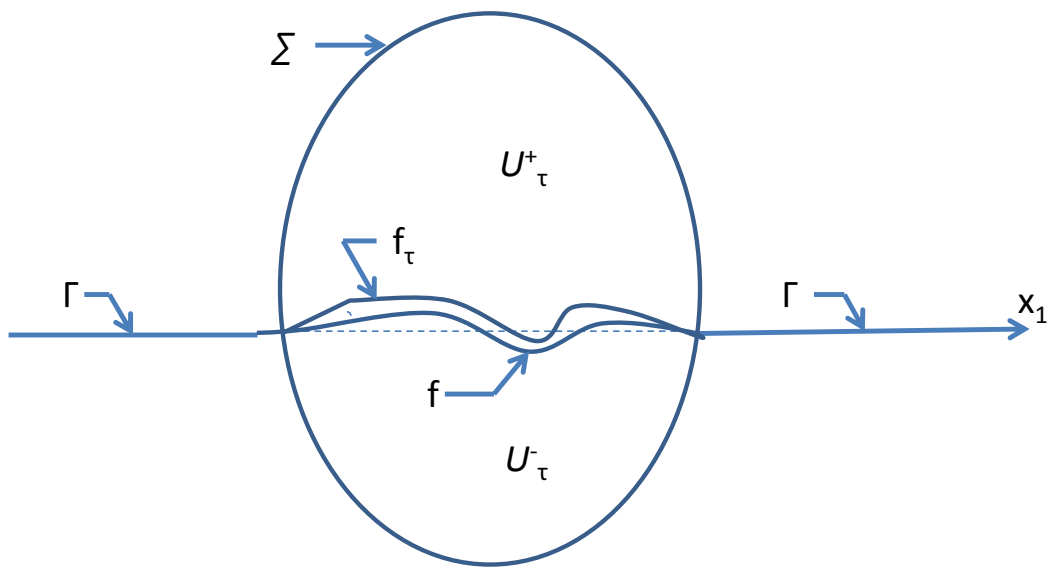

Figure 3: Domain Variation for the Locally Perturbed Surface of Figure 1

for the volume, and

$$
\int_{\Gamma}^{ \pm} \Phi^{ \pm} d s=\int_{\Gamma}\left(\Phi^{+} \pm \Phi^{-}\right) d s
$$

for the surface integral. Also, define a regular function, $u \in \mathcal{U}$, which vanishes on $\Sigma$, and consider the domain functional:

$$
F\left(\mathcal{U}_{\tau}^{+}, \mathcal{U}_{\tau}^{-}\right)=\int_{\mathcal{U}_{\tau}^{ \pm}}^{+}\left(\alpha^{\mp} \mathcal{L}_{\tau}^{ \pm} \psi_{\tau}^{ \pm}\right) u d \vec{x}_{\tau} .
$$

Apply Green's theorem (Eq. (18)) to Eq. (28), and obtain:

$$
\begin{aligned}
F\left(\mathcal{U}_{\tau}^{+}, \mathcal{U}_{\tau}^{-}\right)= & -\int_{\gamma_{\tau}}^{-}\left\{\alpha^{\mp} \psi_{\tau, n}^{ \pm}\right\} u d s_{\tau}- \\
& -\int_{\mathcal{U}_{\tau}^{ \pm}}^{+} \nabla_{\tau}\left(\alpha^{\mp} \psi_{\tau}^{ \pm}\right) \cdot \nabla_{\tau} u d \vec{x}_{\tau}+\int_{\mathcal{U}_{\tau}^{ \pm}}^{+}\left\{\alpha^{\mp}\left(k^{ \pm}\right)^{2}\right\} \psi_{\tau}^{ \pm} u d \vec{x}_{\tau} .
\end{aligned}
$$

$\nabla_{\tau}$ is the gradient with respect to $\vec{x}_{\tau}$, and the negative sign on the $\gamma_{\tau}$ integral is due to the opposing signs of the $\hat{n}$ on the surface. The L.H.S. in Eq. (29) vanishes because of Eqs. (19) and (20). Moreover, since $\alpha^{-} \psi_{\tau, n}^{+}=\alpha^{+} \psi_{\tau, n}^{-}$from the boundary condition in Eq. (22), the surface integral in Eq. (29) vanishes yielding:

$$
-\int_{\mathcal{U}_{\tau}^{ \pm}}^{+} \nabla_{\tau}\left(\alpha^{\mp} \psi_{\tau}^{ \pm}\right) \cdot \nabla_{\tau} u d \vec{x}_{\tau}+\int_{\mathcal{U}_{\tau}^{ \pm}}^{+}\left\{\alpha^{\mp}\left(k^{ \pm}\right)^{2}\right\} \psi_{\tau}^{ \pm} u d \vec{x}_{\tau}=0 .
$$

We now take the Eulerian derivative in Eq. (30), use Eq. (11), and simplify. The result is:

$$
\int_{\mathcal{U}^{ \pm}}^{+}\left[\nabla\left(\alpha^{\mp} \psi^{ \pm \prime}\right) \cdot \nabla u-\alpha^{\mp}\left(k^{ \pm}\right)^{2} \psi^{ \pm \prime} u\right] d \vec{x}=
$$




$$
=\int_{\gamma}^{-}\left[\nabla\left(\alpha^{\mp} \psi^{ \pm}\right) \cdot \nabla u-\alpha^{\mp}\left(k^{ \pm}\right)^{2} \psi u\right] v d s .
$$

We used the fact that $\gamma^{+}=\gamma^{-}=\gamma$, and $\psi^{+}=\psi^{-}=\psi$ on $\gamma$ by the boundary condition in Eq. (3). We now replace $\left(k^{ \pm}\right)^{2} \psi^{ \pm \prime}$ by $-\Delta \psi^{ \pm \prime}$ (cf. Eqs. (23) and (24)) in Eq. (31) to obtain:

$$
\begin{aligned}
\int_{\mathcal{U}^{ \pm}}^{+}\left[\Delta\left(\alpha^{\mp} \psi^{ \pm \prime}\right)\right. & \left.u+\nabla\left(\alpha^{\mp} \psi^{ \pm \prime}\right) \cdot \nabla u\right] d \vec{x}= \\
& -\int_{\gamma}^{-}\left[\nabla\left(\alpha^{\mp} \psi^{ \pm}\right) \cdot \nabla u-\left\{\alpha^{\mp}\left(k^{ \pm}\right)^{2}\right\} \psi u\right] v d s .
\end{aligned}
$$

Applying Green's theorem to the L.H.S. in Eq. (32) yields:

$$
\int_{\mathcal{U}^{ \pm}}^{+}\left[\Delta\left(\alpha^{\mp} \psi^{ \pm \prime}\right) u+\nabla\left(\alpha^{\mp} \psi^{ \pm \prime}\right) \cdot \nabla u\right] d \vec{x}=\int_{\gamma}^{-}\left(\alpha^{\mp} \psi_{, n}^{ \pm \prime}\right) u d s .
$$

The first surface integral in the R.H.S. in Eq. (32) can be evaluated as follows.

$$
\int_{\gamma}^{-}\left[\nabla\left(\alpha^{\mp} \psi^{ \pm}\right) \cdot \nabla u\right] v d s=\int_{\gamma}^{-}\left[\nabla_{\gamma}\left(\alpha^{\mp} \psi^{ \pm}\right) \cdot \nabla_{\gamma} u+\left(\alpha^{\mp} \psi_{, n}^{ \pm}\right) u_{, n}\right] v d s .
$$

As before, $\alpha^{-} \psi_{, n}^{+}=\alpha^{+} \psi_{, n}^{-}$from the boundary condition in Eq. (4). The second integral in the R.H.S. in Eq. (34), therefore, vanishes, yielding:

$$
\begin{aligned}
\int_{\gamma}^{-} & {\left[\nabla\left(\alpha^{\mp} \psi^{ \pm}\right) \cdot \nabla u\right] v d s=} \\
= & \int_{\gamma}^{-}\left[\nabla_{\gamma}\left(\alpha^{\mp} \psi^{ \pm}\right) \cdot \nabla_{\gamma} u\right] v d s \\
= & \left(\alpha^{-}-\alpha^{+}\right) \int_{\gamma}\left\{\left(\nabla_{\gamma} \psi^{+}\right) \cdot\left(\nabla_{\gamma} u\right)\right\} v d s \\
= & \left(\alpha^{+}-\alpha^{-}\right) \int_{\gamma}\left\{\nabla_{\gamma} \cdot\left(v \nabla_{\gamma} \psi^{+}\right)\right\} u d s,
\end{aligned}
$$

in which the identity in Eq. (17) was used. Moreover, $\nabla_{\gamma} \psi^{-}=\nabla_{\gamma} \psi^{+}$. Replace Eqs. (33) and (35) in Eq. (32). This results in:

$$
\int_{\gamma}^{-}\left\{\alpha^{\mp} \psi_{, n}^{ \pm \prime}\right\} u d s=\left(\alpha^{-}-\alpha^{+}\right) \int_{\gamma} \nabla_{\gamma} \cdot\left(v \nabla_{\gamma} \psi^{+}\right) u d s+\int_{\gamma}^{-}\left[\alpha^{\mp}\left(k^{ \pm}\right)^{2} \psi v\right] u d s
$$

from which it follows that:

$$
\alpha^{-} \psi_{, n}^{+\prime}-\alpha^{+} \psi_{, n}^{-\prime}=\left(\alpha^{-}-\alpha^{+}\right)\left\{\nabla_{\gamma} \cdot\left(v \nabla_{\gamma} \psi^{+}\right)\right\}+\left(\alpha^{-} k^{+2}-\alpha^{+} k^{-2}\right) \psi v .
$$

Equation (36) establishes the boundary conditions on $\gamma$ for the normal derivatives, $\psi_{, n}^{ \pm \prime}$, of the derivative fields, $\psi^{ \pm \prime}$. 
Collecting the results in Eqs. (25), (26), (27) and (36) together yields:

$$
\begin{aligned}
\mathcal{L}^{+} \psi^{+\prime}(\vec{x}) & =0, \vec{x} \in \Omega^{+}, \\
\mathcal{L}^{-} \psi^{-\prime}(\vec{x}) & =0, \vec{x} \in \Omega^{-}, \\
\psi^{+\prime}-\psi^{-\prime} & =(\alpha-1) \psi_{, n}^{+} v, \vec{x} \in \gamma, \\
\alpha^{-} \psi_{, n}^{+\prime}-\alpha^{+} \psi_{, n}^{-\prime} & =\left(\alpha^{-}-\alpha^{+}\right) \nabla_{\gamma} \cdot\left(v \nabla_{\gamma} \psi^{+}\right) \\
& +\left(\alpha^{-} k^{+2}-\alpha^{+} k^{-2}\right) \psi^{+} v, \vec{x} \in \gamma, \\
\psi^{+\prime} & =\psi^{-\prime}, \vec{x} \in \Gamma \backslash \gamma, \\
\alpha^{-} \psi_{, n}^{+\prime} & =\alpha^{+} \psi_{, n}^{-\prime}, \vec{x} \in \Gamma \backslash \gamma .
\end{aligned}
$$

The last two boundary conditions in Eqs. (41) and (42) follow simply from the fact that on the flat part of $\Gamma$, the observation points are held constant. Equations (37) through (42) constitute the scattering problem for the Eulerian derivatives of the Helmholtz problem in Eqs. (1)-(4). As can be seen from the above equations, the scattering problem for the derivative fields is essentially the same as that for the unperturbed surface except that the boundary conditions on $\gamma$ for $\psi^{ \pm \prime}$ now depend upon the deformation field through the parameter, $v$. The deformation, therefore, leaves it's imprint via the boundary conditions. The boundary value problem for the derivative fields having been obtained, the Eulerian derivative, $\underline{d} \mathcal{J}$, of the objective function is calculated next. 


\section{The Gradient}

Let $F(\Omega)$ be a real functional of the domain, $\Omega$. Then from Eq. (8), its domain differential, $\underline{d F}(\Omega)$, is:

$$
\underline{d F}(\Omega)=\lim _{\tau \rightarrow 0} \frac{1}{\tau}\left[F_{\tau}\left(\Omega_{\tau}\right)-F(\Omega)\right] .
$$

If the mapping: $\vec{V} \rightarrow \underline{d F}(\Omega)$ is linear and continuous (in $\vec{V}$ ), then $\underline{d F}(\Omega)$ has a gradient, $G_{F}(\Omega, \vec{V})$. In this case, $\underline{d F}(\Omega)$ can be written as:

$$
\underline{d F}(\Omega)=<G_{F}, \vec{V}>_{Y},
$$

where the duality relation $<\cdot>$ is over $Y$, the product space of $G_{F}$ and $\vec{V}$. $G_{F}$ is a distribution which is defined on the boundary, and can be considered to be the gradient of $F(\Omega)$. The objective function was defined in Eq. (5) and its Eulerian derivative in Eqs. (6) and (7). Equation (6) can be written as:

$$
\underline{d} \mathcal{J}(\gamma)=-\operatorname{Re} \int_{\Omega^{+}} \psi^{+\prime} \sum_{m=1}^{M}\left(\Delta^{\delta} \psi^{+}\right)^{*} \delta\left(\vec{x}-\vec{x}_{m}\right) d \vec{x} .
$$

We need to eliminate the derivative field in (45). Towards that let us define an auxiliary field, $p^{+}$, that satisfies the Helmholtz equation below.

$$
\mathcal{L}^{+} p^{+}=-\sum_{m=1}^{M}\left(\Delta^{\delta} \psi^{+}\right)^{*} \delta\left(\vec{x}-\vec{x}_{m}\right) \vec{x} \in \Omega^{+} .
$$

In terms of the field, $p^{+},(45)$ for $\underline{d} \mathcal{J}(\gamma)$ becomes:

$$
\underline{d} \mathcal{J}(\gamma)=\operatorname{Re} \int_{\Omega^{+}} \psi^{+\prime}\left(\mathcal{L}^{+} p^{+}\right) d \vec{x}
$$

Note that $\mathcal{L}^{+}=\Delta+k^{+2}$, and apply Green's theorem to (47). After a straightforward algebra, and upon simplification, we obtain:

$$
\int_{\Omega^{+}} \psi^{+\prime}\left(\mathcal{L}^{+} p^{+}\right) d \vec{x}=\int_{\gamma}\left[p^{+} \psi_{, n}^{+\prime}-\psi^{+\prime} p_{, n}^{+}\right] d \gamma
$$

$\mathcal{L}^{+} \psi^{+\prime}=0$ was used in the above derivation. Substituting for $\psi^{+\prime}$ and $\psi_{, n}^{+\prime}$ from the boundary conditions in Eqs. (39) and (40), respectively, we obtain:

$$
\begin{aligned}
\int_{\Omega^{+}} \psi^{+\prime}\left(\mathcal{L}^{+} p^{+}\right) d \vec{x}= & \int_{\gamma}\left(\frac{1}{\alpha^{-}} P_{, n} p^{+}-P p_{, n}^{+}\right) d \gamma+ \\
& +\int_{\gamma}\left[\frac{1}{\alpha} \psi_{, n}^{-1} p^{+}-\psi^{-1} p_{, n}^{+}\right] d \gamma
\end{aligned}
$$

with

$$
P=(\alpha-1) \psi_{, n}^{+} v
$$


and

$$
P_{, n}=\left(\alpha^{-}-\alpha^{+}\right) \nabla_{\gamma} \cdot\left(v \nabla_{\gamma} \psi^{+}\right)+\left(\alpha^{-} k^{+2}-\alpha^{+} k^{-2}\right) \psi^{+} v
$$

In order to further eliminate the derivative terms in Eq. (49), we define an auxiliary field, $p^{-}$, in $\Omega^{-}$, satisfying the Helmholtz equation:

$$
\mathcal{L}^{-} p^{-}=0, \vec{x} \in \Omega^{-},
$$

and impose the conditions

$$
\begin{aligned}
& p^{-}=p^{+} \text {on } \gamma \\
& p_{, n}^{-}=\alpha p_{, n}^{+} \text {on } \gamma
\end{aligned}
$$

Thus, the auxiliary problem is given by:

$$
\begin{aligned}
\mathcal{L}^{+} p^{+} & =-\sum_{m=1}^{M}\left(\Delta^{\delta} \psi^{+}\right)^{*} \delta\left(\vec{x}-\vec{x}_{m}\right), \vec{x} \in \Omega^{+}, \\
\mathcal{L}^{-} p^{-} & =0, \vec{x} \in \Omega^{-}, \\
p^{-} & =p^{+} \text {on } \gamma, \\
p_{, n}^{-} & =\alpha p_{, n}^{+} \text {on } \gamma .
\end{aligned}
$$

In view of the above, we finally arrive at the following expression for $\underline{d} \mathcal{J}(\gamma)$, namely

$$
\underline{d} \mathcal{J}(\gamma)=-\operatorname{Re} \int_{\gamma}\left(P p_{, n}^{+}-\frac{1}{\alpha^{-}} P_{, n} p^{+}\right) d \gamma
$$

Reducing the surface gradient terms by the Stokes' identity in Eq. (17) then yields:

$$
\begin{aligned}
\underline{d} \mathcal{J}(\gamma)=-\operatorname{Re} \int_{\gamma} d \gamma & {\left[(\alpha-1) p_{, n}^{+} \psi_{, n}^{+}+\right.} \\
+ & \left.\left(1-\frac{1}{\alpha}\right) \nabla_{\gamma} p^{+} \cdot \nabla_{\gamma} \psi^{+}-\frac{1}{\alpha^{-}}\left(\alpha^{-} k^{+2}-\alpha^{+} k^{-2}\right) \psi^{+} p^{+}\right] v,
\end{aligned}
$$

Rewriting Eq. (58) in the form:

$$
\underline{d} \mathcal{J}=\int_{\gamma} G_{\mathcal{J}} v d s
$$

the gradient, $G_{\mathcal{J}}$, is obtained as:

$$
G_{\mathcal{J}}=-\operatorname{Re}\left[(\alpha-1) p_{, n}^{+} \psi_{, n}^{+}+\left(1-\frac{1}{\alpha}\right) \nabla_{\gamma} p^{+} \cdot \nabla_{\gamma} \psi^{+}-\left(k^{+2}-\frac{1}{\alpha} k^{-2}\right) \psi^{+} p^{+}\right]
$$




\section{Applications}

\subsection{EM Scattering}

Thus far, the derivations were carried out in terms of $\psi^{ \pm}$and $\alpha^{ \pm}$, without specifically referring to electromagnetic (EM) scattering. The EM-scattering is considered now. In this case, $\psi$ is the electric $(\vec{E})$ or magnetic $(\vec{H})$ field, and $\alpha^{ \pm}$is to be replaced by $\epsilon$ or $\mu$, the dielectric and the permeability constant, respectively, depending upon the polarization of the incident field. It is well-known [5, 34, 38] that an EM-wave propagation can be described in terms of a TE (transverse electric) and a TM (transverse magnetic) mode (also called perpendicular (s) and parallel (p) polarization in spectroscopy, and VV or $\mathrm{HH}$ in radar work, see, e.g., Cloude [6]). Transverse implies transverse to the plane of the incidence, that is, the plane containing the direction of the wave propagation and the normal to the surface. The two polarizations are schematically illustrated in Figure 4. In the TE mode, $\vec{E}$ is transverse, and in the TM mode, it is $\vec{H}$ that is transverse. Referring to Figure $4, \vec{E}=-\hat{x}_{3} E_{3}$ in TE, and $\vec{H}=-\hat{x}_{3} H_{3}$ in TM. The tangential components of

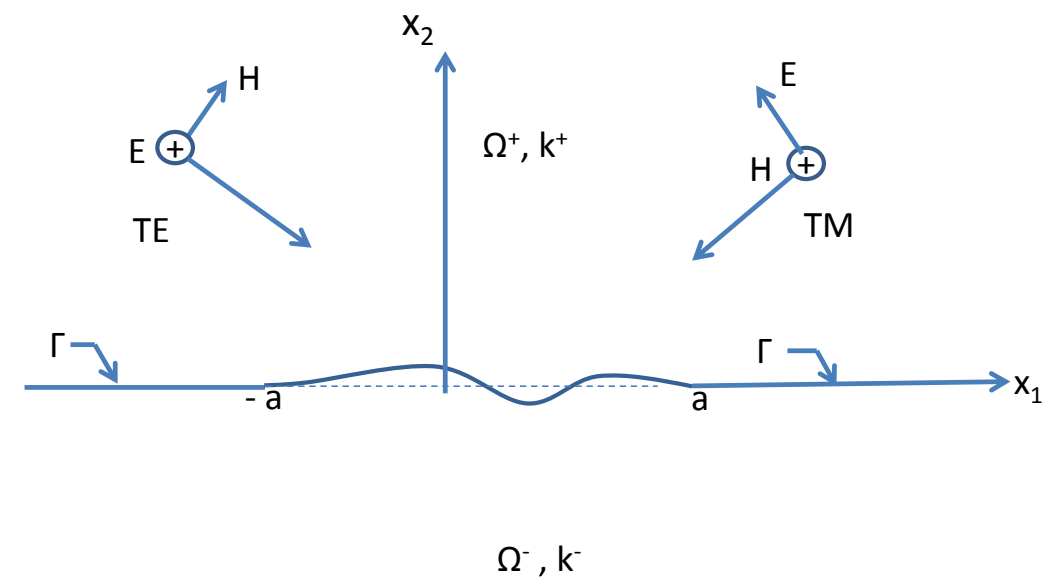

Figure 4: Our Convention of Vector Orientations for TE and TM Polarizations

both $\vec{E}$ and $\vec{H}$ are continuous across the surface. From this and Maxwell's equations, the boundary conditions for the TE mode are:

$$
\begin{aligned}
& E_{3}^{+}=E_{3}^{-}, \\
& E_{3 n}^{+}=E_{3 n}^{-} .
\end{aligned}
$$

Similar conditions for the TM mode are:

$$
\begin{aligned}
H_{3}^{+} & =H_{3}^{-}, \\
\epsilon^{-} H_{3 n}^{+} & =\epsilon^{+} H_{3 n}^{-} .
\end{aligned}
$$


In TE, $\alpha^{ \pm}=1$, whereas in TM, $\alpha^{ \pm}=\epsilon^{ \pm}$. The parameter, $\alpha=\epsilon^{-} / \epsilon^{+}=\epsilon_{r}$, is the relative dielectric constant. With these identifications, Eq. (58) for $\underline{d} \mathcal{J}$ and (59) for $G_{\mathcal{J}}$ can be written down for the two polarizations. For the TE mode:

$$
\begin{aligned}
\underline{d} \mathcal{J}_{T E} & =k^{+2}\left(\epsilon_{r}-1\right) \operatorname{Re} \int_{\gamma} \psi^{+} p^{+} v d s, \\
\overline{\mathbf{G}}_{\mathcal{J}, T E} & =k^{+2}\left(\epsilon_{r}-1\right) \operatorname{Re}\left(\psi^{+} p^{+}\right) .
\end{aligned}
$$

The corresponding expressions for the TM mode are:

$$
\begin{gathered}
\underline{d} \mathcal{J}_{T M}=\operatorname{Re} \int_{\gamma} d s\left[\left(\epsilon_{r}-1\right) p_{, n}^{+} \psi_{, n}^{+}-\left(1-\frac{1}{\epsilon_{r}}\right)\left(\nabla_{\gamma} p^{+} \cdot \nabla_{\gamma} \psi^{+}\right)\right] v \\
G_{\mathcal{J}, T M}=\left(\epsilon_{r}-1\right) p_{, n}^{+} \psi_{, n}^{+}+\left(1-\frac{1}{\epsilon_{r}}\right)\left(\nabla_{\gamma} p^{+} \cdot \nabla_{\gamma} \psi^{+}\right)
\end{gathered}
$$

We will next look at the limiting cases of the gradient results for Neumann and Dirichlet problems.

\subsection{Neumann Surface}

Equation (59) can be used to obtain the gradient for both the Dirichlet and Neumann surface. For the Neumann condition, the first term in the R. H. S. in Eq. (59), i.e., $(\alpha-1) p_{, n}^{+} \psi_{, n}^{+}$, can be set to zero because of the Neumann boundary condition itself. Next, letting the parameter, $\alpha \rightarrow \infty$ in the R. H. S. in Eq. (59) yields:

$$
G_{\mathcal{J}, N}=-\operatorname{Re}\left(\nabla_{\gamma} p^{+} \cdot \nabla_{\gamma} \psi^{+}-k^{+2} \psi^{+} p^{+}\right) .
$$

The subscript $N$ in $G_{\mathcal{J}}$ implies Neumann. This result can be verified by direct calculation as follows.

For the Neumann boundary condition, the unperturbed scattering problems is given by:

$$
\begin{aligned}
\left(\mathcal{L}^{+} \psi^{+}\right)(\vec{x}) & =0, \vec{x} \in \Omega^{+}, \\
\psi^{-}(\vec{x}) & =0, \vec{x} \in \Omega^{-}, \\
\psi_{, n}^{+} & =0 \text { on } \Gamma .
\end{aligned}
$$

The perturbed problem, i.e., $\psi_{\tau}^{+}$, is also given by the above equations. Following the procedures in the preceeding section, the boundary condition for the derivative field is obtained as:

$$
\psi_{, n}^{+\prime}=k^{+2} \psi^{+} v+\nabla_{\gamma} \cdot\left(v \nabla_{\gamma} \psi\right) \text { on } \Gamma,
$$

and the auxiliary problem for $p^{+}$takes the form:

$$
\begin{aligned}
\left(\mathcal{L}^{+} p^{+}\right) & =-\sum_{m=1}^{M}\left(\Delta^{\delta} \psi^{+}\right)^{*} \delta\left(\vec{x}-\vec{x}_{m}\right) \quad \vec{x} \in \Omega^{+}, \\
p_{, n}^{+} & =0 \text { on } \Gamma .
\end{aligned}
$$


By the same reasonings as above, $\underline{d} \mathcal{J}$ and $G_{\mathcal{J}}$ for the Neumann surface are found to be:

$$
\begin{gathered}
\underline{d} \mathcal{J}_{N}=-\operatorname{Re} \int_{\gamma}\left(\nabla_{\gamma} p^{+} \cdot \nabla_{\gamma} \psi^{+}-k^{+2} \psi^{+} p^{+}\right) v d s, \\
G_{\mathcal{J}, N}=-\operatorname{Re}\left(\nabla_{\gamma} p^{+} \cdot \nabla_{\gamma} \psi^{+}-k^{+2} \psi^{+} p^{+}\right) .
\end{gathered}
$$

Equations (68) and (69) are similar to those of Norton [36] for a compactly supported sound-hard obstacle by variational formulation and an augmented objective function. However, there is a sign change in our results from Norton's. It is because the source term in our auxiliary problems for $p^{+}$is opposite to Norton's.

\subsection{Dirichlet Surface}

The same reasonings can be applied to the Dirichlet surface also. In the Dirichlet case, we neglect the terms containing $\nabla \psi^{-}$and $\psi^{+}$in Eq. (58) since by the Dirichlet condition, $\psi^{+}$is identically zero on the surface, Then letting $\alpha \rightarrow 0$ in the remaining term yields the gradient for the Dirichlet surface, namely, $G_{\mathcal{J}, D}=-\operatorname{Re}\left(\psi_{, n}^{+} p_{, n}^{+}\right)$. Again, this result is also verified by direct calculation, as was done for the Neumann problem, but with $\psi^{+}=0, \psi^{+\prime}=-v \psi_{, n}^{+}$in the direct problem, and $p^{+}=0$ in the auxiliary problem. 


\section{Discussion}

Our results for a locally perturbed, infinite surface are found to be similar to the results reported in the literature for compactly supported objects. This is not surprising since the same basic equations apply to either case. The auxiliary field is a purely mathematical construction, and may not have a direct physical meaning. As was pointed out in the text, the source term for the auxiliary field, $p^{+}$, consists of the data misfit functions backpropagated to the scatterer. The auxiliary fields are then the fields generated by this source for the boundary conditions of the problem. A relation between the gradient calculations and reciprocity of the derivative and auxiliary fields can thus be expected. To illustrate that, let us rearrange the equations in a slightly different form.

Let $\mathbf{L}$ be a $2 \times 2$ diagonal matrix whose diagonal elements are $L^{+}$and $L^{-}$, and let $\boldsymbol{\Psi}=\left[\psi^{+}, \psi^{-}\right]^{T}, \boldsymbol{\Psi}^{\prime}=\left[\psi^{+\prime}, \psi^{-\prime}\right]^{T}$, and let $\mathbf{P}=\left[p^{+}, p^{-}\right]^{T}$. Moreover, let $[q]_{\Gamma}$ denote the jump of the quantity, $q$, across the interface. In these notations, Eqs. (1)-(4) become:

$$
\begin{gathered}
\mathbf{L} \Psi=\mathbf{0}, \quad \vec{x} \in R^{2} \backslash \Gamma, \\
{[\psi]_{\Gamma}=0,} \\
{[\alpha \psi, n]_{\Gamma}=0 .}
\end{gathered}
$$

Similarly, Eqs. (37)-(42) take the form:

$$
\begin{gathered}
\mathbf{L} \Psi^{\prime}=\mathbf{0}, \quad \vec{x} \in R^{2} \backslash \Gamma, \\
{\left[\psi^{\prime}\right]_{\Gamma}=P,} \\
{\left[\alpha \psi_{, n}^{\prime}\right]_{\Gamma}=P,_{n},}
\end{gathered}
$$

and the adjoint field Eqs. (53) through (56) are similarly recast as:

$$
\begin{gathered}
\mathbf{L P}=-\operatorname{Re} \sum_{m=1}^{M} \Delta^{\delta *}\left(x_{m}\right) \delta\left(\vec{r}-\vec{x}_{m}\right) \quad \vec{x} \in R^{2} \backslash \Gamma \\
{[p]_{\Gamma}=0} \\
{\left[\alpha p_{, n}\right]_{\Gamma}=0 .}
\end{gathered}
$$

In these notations, Eq. (58) for $\underline{d} \mathcal{J}$ takes the form:

$$
\underline{d} \mathcal{J}=\operatorname{Re} \int_{\Gamma} \mathbf{J} \cdot \mathbf{n} d \Gamma,
$$

where

$$
\mathbf{J}=\frac{1}{\alpha^{-}} p^{+}\left[\alpha \nabla \psi^{\prime}\right]-\left[\psi^{\prime}\right] \nabla p^{+} .
$$

Equation (70) is the Lorentz reciprocity relation between $p^{+}$, and $\psi^{\prime}$ [34]. 
Note that $\mathbf{J}$ does not vanish indicating that the fields are not truly adjoint to each other. The non-vanishing of $\mathbf{J}$ is due to the fact that the forward and reciprocal incident fields do not act on the same scatterer, but the former on the unperturbed and the other on the perturbed scatterer. In other words, $\mathbf{J} \neq \mathbf{0}$ indicates the variation of the reciprocal fields. We have, therefore, used the word auxiliary instead of adjoint. It may be interesting to point out that the usual picture of reciprocity is Helmholtz's reciprocity in which the reciprocity is between a point source and a point observer. However, Helmholtz's reciprocity does not always hold for sources and observers of finite extent [21]. Lorentz's reciprocity, on the other hand, is an integral relation over the sources. 


\section{Conclusion}

The inverse scattering solution for the shape and/or material parameters of a scattering object is often posed as a problem in nonlinear optimization in which an objective function is minimized with respect to $N$ (usually large) number of unknown parameters characterizing the scatterer. The minimization is often performed iteratively where the gradient of the objective function in the parameter vector is required in every stage of iteration. The application of the domain differentiation technique provides a highly efficient solution to the problem. This report gives a detailed account of the domain derivative calculation of the gradient for a one-dimensional, locally perturbed infinitely long dielectric surface. Unlike the so-called adjoint field method which applies variational procedures to an objective function augmented by the reduced wave equation via a Lagrange multiplier, the method in this report is purely algebraic in nature in that it evaluates the derivative by applying domain differentiaion directly to the scattering equations without any other intervening mathematical formalism. The calculations presented here are straightforward and easy to follow. Moreover, the mathematical transformation of the scattering problem into the corresponding problem for the differentiated fields can be visualized explicitly. The solution requires an auxiliary scattering problem. The formulation of and the motivation behind the auxiliary problem are explicitly demonstrated. The gradients are calculated

for the electromagnetic TE and TM scattering from a dielectric surface as also for scale wave scattering from a Neumann and a Dirichlet surface. Finally, the relationship of the results thus obtained with the Lorentz reciprocity is discussed. 


\section{References}

[1] S. Afifi and M. Diaf. Scattering by random rough surfaces: Study of direct and inverse problem. Optics Commun., 265:11-17, 2006.

[2] T. Arens and T. Hohage. On radiation condition for rough surface scattering problems. IMA J. Appl. Math., 70:839-847, 2005.

[3] F. G. Bass and I. M. Fuks. Wave Scattering from Statistically Rough Surfaces. Pergamon, Oxford, 1979.

[4] P. Beckmann and A. Spizzichino. The Scattering of Electromagnetic Waves from Rough Surfaces. Artech House, Norwood, Massachusetts, 1987.

[5] M. Born and E. Wolf. Principles of Optics. Cambridge University Press, Cambridge, U. K., 7th edition, 1999.

[6] S. Cloude. Polarization. Oxford University Press, Oxford, UK, 2010.

[7] D. Colton and R. Kress. Inverse Acoustic and Electromagnetic Scattering. Springer, Berlin, 1992.

[8] N. Dechamps, N. de Beaucoudrey, C. Bourlier, and S. Toutain. Fast numerical method for electromagnetic scattering by rough surface layered interfaces: Propagationinside-layer expansion method. J. Opt. Soc. Am. A, 23:355-369, 2006.

[9] J. A. DeSanto and G. S. Brown. Analytical techniques for multiple scattering from rough surfaces. In E. Wolf, editor, Progress in Optics XXIII, pages 1-62. NorthHolland, Amsterdam, Netherlands, 1986.

[10] J. A. DeSanto and P. Martin. On the derivation of boundary integral equations for scattering by an infinite one-dimensional rough surface. J. Acoust. Soc. Am., 102:67-77, 1997.

[11] O. Dorn. Shape reconstruction in scattering media with voids using a transport model and level sets. Canad. Appl. Math. Quart., 10:239-275, 2002.

[12] O. Dorn, E. L. Miller, and C. M. Rappaport. A shape reconstruction method for electromagnetic tomography using adjoint fields and level set. Inverse Problems, 16:1119-1156, 2000.

[13] H. W. Engl, M. Hanke, and A. Neubauer. Regularization of Ill-posed Problems. Kluwer, Dordrecht, 1996.

[14] G. R. Feijoo. Reconstruction of periodic structures from optical scattering measurements using adjoint equations. J. Opt. Soc. Am. A, 25:1906-1920, 2008. 
[15] V. B. Glasko. Inverse Problems of Mathematical Physics. Springer-Verlag, New York, 1989.

[16] C. Groetsch. Inverse Problems in the Mathematical Sciences. Vieweg, Braunschweig, 1993.

[17] H. Gross, A. Rathsfeld, F. Schoze, M. Bar, and U. Dersch. Optimal sets of measurement data for profile reconstruction in scatterometry. Proc. of SPIE, 6617:66171B1-66171B-12, 2007.

[18] E. J. Haug and J. Cea. Optimization of Distributed Parameter Structures, volume II. Sijhoff and Noordhoff, Nijhoff, The Hague, 1981.

[19] E. J. Haug, K. K. Choi, and V. Komkov. Design Sensitivity Analysis of Structural Systems. Academic Press, Orlando, 1986.

[20] D. Henry. Perturbation of the Boundary in Boundary - Value problems of Partial Differential Equations, volume 318 of Lecture Notes Series. London Mathematical Society, London, UK, 2005.

[21] B. J. Hoenders. On the invalidity of helmholtz's receiprocity theorem for green's functions describing the propagation of scalar wave field in a non empty- and empty space. Optik, 54:373-380, 1980.

[22] P. Hursky, M. B. Porter, B. D. Cornuelle, W. S. Hodgkiss, and W. A. Kuperman. Adjoint modeling for acoustic inversion. J. Acoust. Soc. Am., 115:607-619, 2004.

[23] Y. Q. Jin. Theory and Applications of Information Retrievals From Electromagnetic Scattering And Remote Sensing. Springer, Dordrecht, 2006.

[24] D. A. Kapp and G. S. Brown. A new numerical method for rough surface scattering calculations. IEEE Trans. Ant. Propagat., 44:711-722, 1996.

[25] O. D. Kellogg. Foundations of Potential Theory. Springer, New York, 1929.

[26] A. Kirsch. The domain derivative and two applications in inverse scattering theory. Inverse Problems, 9:81-96, 1993.

[27] A. Kirsch. An Introduction to The Mathematical Theory of Inverse Problems. Springer-Verlag, New York, 1996.

[28] R. Kress. Inverse scattering for a locally perturbed half-plane. Inverse Problems, 16:1541-1559, 2000.

[29] D. G. Luenberger. Optimization by Vector Space Methods. Wiley, New York, 1969. 
[30] C. Lukianowicz. Direct and inverse problem in scatterometry of rough surfaces. Proc. SPIE, 4517:120-125, 2001.

[31] P. R. McGillivary and D. W. Olderburg. Methods for calculating frechet derivatives and sensitivities for non-linear inverse problems: a comparative study. Gephys. Prospect., 38:499-524, 1990.

[32] N. Morita, N. Kumagai, and J. R. Mautz. Integral Equation Methods For Electromagnetics. Artech House, Boston, 1990.

[33] J. M. Nédélec. Acoustic and Electromagnetic Equations. Springer, New York, 2001.

[34] M. Nieto-Versperinas. Scattering And Diffraction In Physical Optics. Wiley, New York, 1997.

[35] S. J. Norton. Iterative inverse scattering algorithms: Methods for computing frechet derivative. J. Acoust. Soc. Am., 106:2653-2660, 1999.

[36] S. J. Norton. Iterative algorithms for computing the shape of a hard scattering object: Computing the shape derivative. J. Acoust. Soc. Am., 116:1002-1008, 2004.

[37] J. A. Ogilvy. Theory of Wave Scattering from Random Rough Surfaces. A. Hilger, Bristol, England, 1991.

[38] A. F. Peterson, S. L. Ray, and R. Mittra. Computational Methods For Electromagnetics. Oxford University Press, Oxford, 1998.

[39] S. O. Rice. Reflection of elelctromagnetic waves from slightly rough surfaces. Commun. Pure Appl. Math., 4:351-378, 1951.

[40] M. Saillard and D. Maystre. Scattering from metallic and dielectric rough surfaces. J. Opt. Soc. Am. A, 7:982-990, 1999.

[41] M. Saillard and G. Toso. Electromagnetic scattering from bounded or infinite subsurface bodies. Radio Sci., 32:1347-1359, 1997.

[42] M. Stankova, J. Burov, and M. Burova. Computer reconstruction of a periodic surface by means of the scattered and transmitted acoustic fields. Meas. Sci. Technol., 12:1330-1335, 2001.

[43] A. Thode and K. Kim. Multiple-order derivatives of a waveguide acoustic field with respect to sound speed, density and frequency. J. Acoust. Soc. Am., 116:3370-3383, 2004.

[44] E. I. Thorsos. Acoustic scattering from 'piarson-moskowitz' sea surface. J. Acoust. Soc. Am., 83:78-92, 1988. 
[45] L. Tsang, J. A. Kong, K-H Ding, and Ao. Scattering of Electromagnetic Waves: Numerical Simulations, volume II. Wiley, New York, 2001.

[46] A. G. Voronovich. Wave Scattering from Rough Surfaces. Springer-Verlag, Berlin, 1999.

[47] R. J. Wombell and J. A. DeSanto. Reconstruction of rough-surface profiles with the kirchhoff approximation. J. Opt. Soc. Am. A, 8:1892-1897, 1991.

[48] R. J. Wombell and J. A. DeSanto. The reconstruction of shallow rough surface profiles from scattered field data. Inverse Probl., 7:L7-L12, 1991.

[49] J-P. Zolesio. Material derivative (or speed) method of shape optimization. In $O p$ timization of Distributed Parameter Structures, volume II, pages 1089-1151. Sijhoff and Nordhoff, Nijhoff, The Hague, 1981. 


\section{List of Acronyms, Abbreviations, and Symbols}

$\begin{array}{ll}\text { Acronym } & \text { Description } \\ f & \text { rough interface } \\ k^{+} & \text {Wave number of upper half space } \\ k^{-} & \text {Wave number of lower half space } \\ \underline{d} F & \text { Domain differential of } F \\ \text { TE } & \text { Tranverse Electric } \\ \text { TM } & \text { Transverse Magnetic }\end{array}$

